\title{
Increased Frequency of Guillain-Barré Syndrome in HIV Infection: A Prospective Cohort Study
}

Franclo Henning* and Patrick Bouic

Faculty of Medicine and Health Sciences, Stellenbosch University, Tygerberg, Western Cape, South Africa

"Corresponding author: Franclo Henning, Faculty of Medicine and Health Sciences, Stellenbosch University, Tygerberg, Western Cape, South Africa, Tel: 27 79 5240851; Fax: 2786 6087654; E-mail: fhenning@sun.ac.za

Copyright: $\odot 2014$ Henning F, et al. This is an open-access article distributed under the terms of the Creative Commons Attribution License, which permits unrestricted use, distribution, and reproduction in any medium, provided the original author and source are credited.

Received date: May 15, 2014; Accepted date: August 01, 2014; Published date: August 10, 2014

\section{Abstract}

Background: Guillain-Barré syndrome (GBS) has been reported in HIV-positive individuals, but the incidence and characteristics in this group of patients has not systematically been investigated beyond case reports and retrospective series. The aim of this study was to compare the incidence and characteristics of GBS in HIV positive and -negative individuals.

Methods: We performed a prospective, comparative study over a $3 \frac{1}{2}$ year period in the Western Cape province of South Africa. All adult patients with GBS were included and classified into 2 groups based on HIV status. The two groups were compared with regards to clinical, electrophysiological and laboratory features. Patients were followed until stable or recovered, for a maximum of 12 months.

Results: 28 patients were included in the study, of which 15 were HIV-positive. Using estimated HIV prevalence data for the same geographical area during the study period, the incidence of GBS in HIV-positive patients was calculated to be $18.74,95 \% \mathrm{Cl}[7.69,40.60]$ times higher than in HIV-negative patients. Except for the frequency of hyponatraemia, there were no statistically significant differences between the 2 groups with regards to presenting features, severity of illness, GBS subtypes, and treatment response. GBS occurred in all stages of HIV infection, and was the presenting feature of HIV infection in 13 patients.

Interpretation: The incidence of GBS is strikingly increased in HIV infection. The reason for this is still uncertain, but can probably be explained by immune dysregulation. HIV infection does not appear to influence the short term outcome of GBS.

Keywords: Guillain-Barrésyndrome; Acuteinflammatory demyelinating polyneuropathy; Acute inflammatory polyneuropathy; HIV; AIDS

\section{Introduction}

Guillain-Barré syndrome (GBS) is an acute inflammatory condition affecting peripheral nerves and nerve roots, often preceded by a flulike illness or gastroenteritis [1,2]. Rapidly progressive generalized weakness is the hallmark, and $17-30 \%$ of cases develop severe respiratory weakness requiring ventilator support [3]. Treatment consists of intravenous immunoglobulin (IVIg) or plasma exchange (PE), and the majority of patients recover well [4]. However, despite advances in intensive care, the mortality rate is still $4-15 \%$, and up to $20 \%$ of survivors are permanently disabled [5]. The incidence of GBS is between 1.2 and 1.9 per 100000 [5-8]. However, because this informationis derived from populations with a low prevalence of HIV infection, it does not provide information about the incidence of GBS in HIV-infected individuals. Although GBS has been reported in HIVpositive individuals [9-12] and is regarded as more frequent in this group [13], data beyond case reports and small case series is scarce. Furthermore, although the presentation and course of GBS is thought to be similar in HIV-positive and -negative an individual, no systematic investigation has been performed to explore this.
We performed a prospective study to compare the epidemiology, presentation and course of GBS between HIV-positive and -negative patients. Additionally, we aimed to characterize the profile of HIVpositive patients who develop GBS with regards to CD4+ count, concomitant opportunistic infections, and the use of anti-retroviral treatment.

\section{Patients and Methods}

\section{Patients}

We performed a prospective, observational study to detect all cases of Guillain-Barré syndrome in HIV-positive and -negative individuals in Tygerberg Hospital, a tertiary referral centre in the Western Cape province of South Africa, for a 31/2 year period (July 2008 to December 2011). Patients were included if they were 18 years old, met accepted criteria for GBS [14], and presented within 4 weeks of symptom onset. Patients with pre-existing serious systemic or neurological disease that could complicate the diagnosis or course of GBS were excluded, as well as patients who were unable or unwilling to attend follow-up at Tygerberg Hospital. In the public healthcare system in the Western Cape, specialist neurology and electrodiagnostic services are only available in tertiary hospitals. Consequently, all potential cases of GBS requiring diagnostic workup are referred to tertiary hospitals. Referrals 
Page 2 of 5

would include a spectrum of patients ranging from those requiring ICU care to less complicated and less severely affected cases.

Thirty nine patients diagnosed with GBS were screened during the study period. Eleven patients were excluded for the following reasons: 3 declined participation, 5 were unable to provide informed consent because they were intubated and ventilated, 1 died shortly after admission, 1 had a serious pre-existing disease, and 1 was unable to attend follow-up. Of the 28 patients included in the study, 15 (54\%) were HIV-positive. Of the excluded patients, 5 were HIV-positive and 6 were HIV-negative.

After inclusion in the study, patients were managed according to standard practices at our institution. Patients who were unable to walk unassisted were treated with either plasma exchange (PE) or intravenous immunoglobulin (IVIg), according to guidelines [15]. The study was approved by the Human Research Ethics Committee of the Faculty of Medicine and Health Sciences, Stellenbosch University, and has been performed in accordance with the 1964 Declaration of Helsinki and its later amendments.

\section{Assessments}

A thorough disease history was obtained and all patients underwent a detailed clinical evaluation. Disease severity was assessed by means of the GBS disability and arm scales, [16] and the MRC sum score. The MRC sum score is a summation of the MRC grades (0-5) of the following muscle pairs: shoulder abductors, elbow flexors, wrist extensors, hip flexors, knee extensors, and ankle dorsiflexors. The sum score ranges from 0 (total paralysis) to 60 (normal strength).

Bilateral nerve conduction studies, including motor-, sensory- and F-responses were performed on all patients. Motor- and F-responses were investigated in the median, ulnar, common peroneal and posterior tibial nerves. Sensory studies included median palmar, ulnar palmar and sural responses. For each patient, results obtained were classified based on accepted electrophysiological classification criteria for GBS [17]. Diagnostic categories include normal, primary demyelinating, primary axonal, unexcitable and equivocal. Additionally, abnormal studies were further classified according to modalities involved (motor, sensory or both). These categories, in combination with clinical information (absence or presence of sensory abnormalities), were then used to classify individual patients into one of three subtypes of Guillain-Barré syndrome, namely acute inflammatory demyelinating polyneuropathy (AIDP), acute motor and sensory axonal neuropathy (AMSAN) or acute motor axonal neuropathy (AMAN), or undetermined if criteria for none of these were met. HIV testing was performed and patients were divided into two groups according to serostatus. Results of CSF analysis were available for $13 \mathrm{HIV}$-positive and $11 \mathrm{HIV}$-negative patients. In addition, the stage of HIV-infection, CD4+ count and the presence of opportunistic infections were also assessed in HIV-positive patients.

\section{Statistical analysis}

The two patient groups were compared with regards to clinical, laboratory and electrophysiological variables. Data was analysed using Microsoft Excel 2010. Differences in metric variables between the two groups were tested using the independent $\mathrm{t}$-test (two-tailed), while differences in categorical variables were tested using Fisher's exact test. $\mathrm{P}$-values less than 0.05 were regarded as significant.

\section{Follow up}

Patients were followed up every threemonths until (1) complete recovery, or (2) no further improvement at two consecutive visits or, (3) one year from admission. At follow-up, patients were reassessed by means of the GBS disability scale, GBS arm scale, and the MRC sum score.

\section{Results}

\section{Comparison between the HIV-positive and-negative groups at presentation}

A comparison of the clinical and laboratory features between the two groups at the time of presentation to hospitalis provided in Table 1. There were no statistically significant differences between the two groups except for the number of patients with serum hyponatraemia, which was more commonly noted in the HIV-positive group. Of the ten HIV-positive patients who reported a preceding infection, 7 had a respiratory tract infection and 3 had a diarrhoeal illness, whereas 3 of the $8 \mathrm{HIV}$-negative patients reported a respiratory tract infection and 5 a diarrhoeal illness. This difference was not statistically significant. Eleven (73\%) of the HIV-positive patients were diagnosed with AIDP and 1 (7\%) with AMAN. In the three remaining patients in the HIVpositive group, the subtype could not be determined because the conduction studies were either normal (2 patients) or equivocal (1 patient). Of the HIV-negative patients, 10 patients (77\%) were diagnosed with AIDP, 1 (8\%) with AMAN and 2 (15\%) with AMSAN.

\begin{tabular}{|l|l|l|}
\hline & $\begin{array}{l}\text { HIV-positive } \\
(\mathbf{n = 1 5})\end{array}$ & $\begin{array}{l}\text { HIV-negative } \\
\text { (N=13) }\end{array}$ \\
\hline Clinical features & & \\
\hline Median age [years] (range) & $42(26-65)$ & $46(19-78)$ \\
\hline Male & $11(73 \%)$ & $10(77 \%)$ \\
\hline Symptomatic preceding infection & $10(67 \%)$ & $8(62 \%)$ \\
\hline $\begin{array}{l}\text { Median MRC sum score at nadir } \\
\text { (range) }\end{array}$ & $32(12-54)$ & $38(2-54)$ \\
\hline $\begin{array}{l}\text { Median GBS disability grade at nadir } \\
\text { (range) }\end{array}$ & $4(3-5)$ & $4(2-5)$ \\
\hline Median arm grade at nadir (range) & $2(1-3)$ & $2(1-4)$ \\
\hline Mean time to nadir in days (range) & $10(2-19)$ & $9(2-27)$ \\
\hline Laboratory features & & $2(18 \%)[n=11]$ \\
\hline Demyelinating neuropathy (AIDP) & $11(73 \%)$ & $10(77 \%)$ \\
\hline Serum hyponatraemia (<135mmol/l) & $9(60 \%)$ & $\begin{array}{l}1.49(0.42-2.69) \\
{[n=13]}\end{array}$ \\
\hline Mean CSF protein [g/l] (range) & $5(38 \%)[n=13]$ & $26.84)$ \\
\hline CSF lymphocytosis (>5 cells / mm3) & (18) & \\
\hline
\end{tabular}

Table 1. Clinical and laboratory features of HIV-positive $(n=15)$ and negative $(\mathrm{n}=13)$ patients. Where assessments / tests were not performed in all patients, the number of patients $(n)$ that did undergo the test / assessment is stated in brackets ${ }^{*}=\mathrm{p}<0.05$ (significant). 


\section{Characteristics of HIV-positive patients at the time of presentation}

Of the 15 HIV-positive patients, 13 were diagnosed as being infected with HIV at the time of presentation with GBS. Neither of the two patients known with HIV-infection was taking anti-retroviral treatment (ART). The median CD4+ count in the HIV-positive group was 270 cells $/ \mathrm{mm}^{3}$ (range 32-656). Four patients had CD4+ counts less than 200 cells $/ \mathrm{mm}^{3}$ (of which 2 were less than 50 cells $/ \mathrm{mm}^{3}$ ), seven patients had counts between 200 and 350 cells $/ \mathrm{mm}^{3}$, and four patients had CD4+ counts greater than 350 cells $/ \mathrm{mm}^{3}$. According to the CDC classification system, 5 patients had AIDS (4 had concomitant infection (tuberculosis), and one had a CD4+ count $<200$ cells $/ \mathrm{mm}^{3}$ ). Thirteen patients underwent lumbar puncture, of whom 5 (38\%) had a CSF lymphocytic pleocytosis (range 18-40 cells $/ \mathrm{mm}^{3}$ ).

\section{Incidence of GBS in HIV-positive patients}

Since the study was not community-based, the absolute incidence of GBS in the HIV-positive population could not be determined. However, the incidence of GBS in the HIV-positive population relative to the HIV-negative population can be calculated based on the estimated prevalence of HIV infection in the general population from which the study sample originates. Based on UNAIDS SPECTRUM estimates, the prevalence for the Western Cape Province was $6.2 \%$, $6.2 \%, 6.16 \%$ and $4.75 \%$ (mean $=5.8 \%$ ) for the years $2008-2011$ (available on www.doh.gov.za). If the incidence of GBS was similar in the HIV-positive and -negative populations, approximately $5.8 \%$ of GBS cases would be expected to be HIV-positive. However, the actual number of GBS cases with HIV infection in our study was $53.6 \%$ (15/28). This represents a 9.2-fold enrichment of HIV-seropositive patients in our cohort of GBS. Even when screened patients who were excluded from the study are included in the calculation, there remains an 8.8-fold enrichment. If the average HIV-seroprevalence rate of 5.8\% is used, the incidence of GBS in our population is calculated to be $18.74,95 \%$ CI $[7.69,40.60]$ times higher in HIV-positive than in HIVnegative individuals.

\section{Treatment and course}

Eight (53\%) of HIV-positive and 7 (54\%) of HIV-negative patients required treatment with PE or IVIg, and two patients in each group required intubation and mechanical ventilation. Two patients in the HIV-positive group experienced secondary worsening after initial improvement, and one of this required re-treatment. No patients in the HIV-negative group experienced secondary worsening. In the HIV-positive group, 4 patients (27\%) were diagnosed with pulmonary tuberculosis during admission, and 1 patient developed pulmonary tuberculosis 4 months after admission with GBS. One patient in the HIV-negative group was diagnosed with bronchus carcinoma. Two patients in each group died: both in the HIV-positive group from hospital-acquired pneumonia (CDC stages $\mathrm{A} 2$ and $\mathrm{C} 2$ respectively), and 1 each from bronchus carcinoma and cardiac arrest in the HIVnegative group. The mean duration of hospital stay (days) for survivors was 36 days in the HIV-positive group and 26 days in the HIV-negative group ( $\mathrm{p}=0.34)$.

After discharge, survivors were followed for up to 12 months (mean $=4$ months). Over this period, 9 of the $13 \mathrm{HIV}$-positive and 3 of the $11 \mathrm{HIV}$-negative patients were lost to follow-up. All patients who were followed either improved or recovered completely. Due to the large amount of patients lost to follow-up, reliable conclusions about the long-term outcomes were not possible. However, no patients were re-referred with recurrent weakness, which would suggest chronic inflammatory demyelinating polyneuropathy (CIDP) rather than GBS.

\section{Discussion}

The association between GBS and HIV infection has been reported frequently over the last 25 years, mainly in the form of case reports and series. Despite this, the magnitude of the association between the two disorders has not been quantified satisfactorily. A retrospective study from Zimbabwe reported that 16 of 29 patients (55\%) with GBS had concomitant HIV infection, while the seroprevalence of HIV infection in Zimbabwe was estimated at $4.3 \%$ at the time [18]. In our prospective cohort of 28 patients with GBS, we found a more than nine fold higher HIV-seropositivity rate than expected and calculated the incidence to be at least 18 times higher in HIV-positive individuals.

The relation between GBS and the stage of HIV infection is also unclear. Although most authors describe GBS as a manifestation of early HIV infection or even seroconversion, numerous reports of GBS in advanced immunosuppression are available, with CD4+ counts as low as 4 cells $/ \mathrm{mm}^{3}$ [9-11]. In our cohort, one third of HIV-positive patients had AIDS. Interestingly, in 13 (87\%) of the 15 patients, GBS was the presenting feature of HIV infection. Clinical, laboratory and electrophysiological features were similar to the HIV-negative group. Although a statistically significant difference in the proportion of patients with serum hyponatraemia (presumably related to inappropriate $\mathrm{ADH}$ secretion) was found, this is likely to be related to a lower than expected proportion in the HIV-negative group, as compared to previously published data [19]. It is noteworthy that, despite the underlying immunosuppression, the short term outcome of the disorder appears to be similar in the HIV-positive and -negative groups, although the sample size does not allow firm conclusions to be drawn.

Our study has some limitations. Firstly, the sample size does not allow a reliable comparison of the two groups and small differences may have been missed. However, due to the scarcity of GBS, a sufficiently large sample size may take many years to obtain. Secondly, because the study was not community based, no conclusions about the actual incidence of GBS in HIV-infected patients can be drawn. However, we believe that our findings do suggest a meaningful increase in the incidence of GBS in HIV-infected compared to noninfected individuals. Thirdly, the poor retention of participants in the study precludes meaningful analysis and comparison of the long-term outcome. Lastly, since no HIV-positive patients were taking ART, the generalizability of our results to populations with a higher rate of ART use is uncertain, as ART could theoretically protect against the development of immune-mediated conditions by decreasing immune dysregulation [20].

The development of autoimmune conditions in immunodeficientpatients, although seemingly counterintuitive, is now a well-recognized phenomenon [21,22]. Immune dysregulation in HIV may involve $\mathrm{T}$ or $\mathrm{B}$ cells, or both, and may lead to autoimmune phenomena unique to HIV or to classical autoimmune syndromes [23]. Immune dysregulation is probably related to chronic immune activation accompanying HIV infection, likely induced and maintained by translocation of microbial products across the intestinal mucosa into the systemic circulation, probably mediated by depletion of Th17 cells in the gut mucosa [24]. Another mechanism likely to be 
involved in chronic immune activation is homeostatic peripheral expansion (HPE). This process occurs during lymphopenia and describes an augmented T-cell proliferation to both foreign antigens (cognate and gut-associated commensal organisms) and self-antigens. However, because auto-immunity does not develop in all lymphopenic individuals, it is reasonable to assume that lymphopenia alone is not sufficient to induce auto-immunity. Some authors have proposed a "two-hit model" to account for the loss in self-tolerance underpinning auto-immunity [25]. According to this theory, the chronic immune activation observed in lymphopenia provides a fertile environment for a "second hit", such as cytokine overproduction or localized inflammation, to induce auto-immune disease. In GBS, such a second hit could conceivably be a flu-like syndrome or diarrhoeal illness, exposing the activated immune system to a foreign antigen and thereby inducing auto-immune disease by means of molecular mimicry, widely accepted as a pathomechanism in GBS [26]. In this respect, GBS differs from most other autoimmune disorders described in HIV, as the commonly desribed disorders are not typically regarded as related to molecular mimicry triggered by a foreign antigen $[21,22]$. An interesting question is whether the triggering epitope is a component of an opportunistic infection or the HIV virus itself, as described in HIV-related anaemia for example [27].

In addition to above-mentioned systemic immune dysregulation, HIVinfected individuals may also be more vulnerable to developing auto-immune diseases involving the central (CNS) and peripheral nervous system (PNS). Normally, neurons in the CNS and PNS are shielded from systemic inflammatory reactions and immune responses by the blood-brain barrier (BBB) and blood-nerve barrier (BNB), respectively. Malfunction of these barriers causes leakage of immunoglobulins and cytokines, which are not allowed through under normal circumstances, into the endoneurial space. In the case of GBS, this disruption of the $\mathrm{BNB}$ is considered paramount in the development of the disorder, and has been shown to be induced by sera from patients with GBS [28]. BBB function has also been shown to be altered by HIV-infection, both in vivo and in vitro [29]. Whether the same is true for the BNB remains to be investigated, but, if indeed the case, predisposes the PNS to immune-mediated damage, as occurs in GBS.

In summary, we have shown that the incidence of GBS is substantially increased in HIV-positive compared to -negative individuals. GBS can occur in any stage of HIV infection, and is often the presenting manifestation. The short-term outcome of HIVassociated GBS appears similar to that of GBS in HIV-seronegative patients, but the longer-term outcome remains to be investigated. The increased incidence of GBS in HIV-infected individuals is probably related to immune dysregulation, the molecular mechanisms of which warrants further investigation.

\section{References}

1. Winer JB, Hughes RA, Anderson MJ, Jones DM, Kangro H, et al. (1988) A prospective study of acute idiopathic neuropathy. II. Antecedent events. J Neurol Neurosurg Psychiatry 51: 613-618.

2. Guillain-Barre Syndrome Study Group (2000) Guillain-Barré syndrome: an Italian multicentre case-control study. Guillain-Barré Syndrome Study Group. Neurol Sci 21: 229-234.

3. Hughes RA, Wijdicks EF, Benson E, Cornblath DR, Hahn AF, et al. (2005) Supportive care for patients with Guillain-Barré syndrome. Arch Neurol 62: 1194-1198.
4. Hughes RA, Swan AV, Raphaël JC, Annane D, van Koningsveld R, et al. (2007) Immunotherapy for Guillain-Barré syndrome: a systematic review. Brain 130: 2245-2257.

5. Rees JH, Thompson RD, Smeeton NC, Hughes RA (1998) Epidemiological study of Guillain-Barré syndrome in south east England. J Neurol Neurosurg Psychiatry 64: 74-77.

6. Bogliun G, Beghi E; Italian GBS Registry Study Group (2004) Incidence and clinical features of acute inflammatory polyradiculoneuropathy in Lombardy, Italy, 1996. Acta Neurol Scand 110: 100-106.

7. Chiò A, Cocito D, Leone M, Giordana MT, Mora G, et al. (2003) Guillain-Barré syndrome: a prospective, population-based incidence and outcome survey. Neurology 60: 1146-1150.

8. Govoni V, Granieri E (2001) Epidemiology of the Guillain-Barré syndrome. Curr Opin Neurol 14: 605-613.

9. Brannagan TH 3rd, Zhou Y (2003) HIV-associated Guillain-Barré syndrome. J Neurol Sci 208: 39-42.

10. Qureshi AI, Cook AA, Mishu HP, Krendel DA (1997) Guillain-Barré syndrome in immunocompromised patients: a report of three patients and review of the literature. Muscle Nerve 20: 1002-1007.

11. Parry O, Mielke J, Latif AS, Ray S, Levy LF, et al. (1997) Peripheral neuropathy in individuals with HIV infection in Zimbabwe. Acta Neurol Scand 96: 218-222.

12. Cornblath DR, McArthur JC, Kennedy PG, Witte AS, Griffin JW (1987) Inflammatory demyelinating peripheral neuropathies associated with human T-cell lymphotropic virus type III infection. Ann Neurol 21: 32-40.

13. Verma A (2001) Epidemiology and clinical features of HIV-1 associated neuropathies. J Peripher Nerv Syst 6: 8-13.

14. Asbury AK, Cornblath DR (1990) Assessment of current diagnostic criteria for Guillain-Barré syndrome. Ann Neurol 27 Suppl: S21-24.

15. Hughes RA, Wijdicks EF, Barohn R, Benson E, Cornblath DR, et al. (2003) Practice parameter: immunotherapy for Guillain-Barré syndrome: report of the Quality Standards Subcommittee of the American Academy of Neurology. Neurology 61: 736-740.

16. (1997) Randomised trial of plasma exchange, intravenous immunoglobulin, and combined treatments in Guillain-Barré syndrome. Plasma Exchange/Sandoglobulin Guillain-Barré Syndrome Trial Group. Lancet. 349: 225-230.

17. Hadden RD, Cornblath DR, Hughes RA, Zielasek J, Hartung HP, et al. (1998) Electrophysiological classification of Guillain-Barré syndrome: clinical associations and outcome. Plasma Exchange/Sandoglobulin Guillain-Barré Syndrome Trial Group. Ann Neurol 44: 780-788.

18. Thornton CA, Latif AS, Emmanuel JC (1991) Guillain-Barré syndrome associated with human immunodeficiency virus infection in Zimbabwe. Neurology 41: 812-815.

19. Saifudheen K, Jose J, Gafoor VA, Musthafa M (2011) Guillain-Barre syndrome and SIADH. Neurology 76: 701-704.

20. Lederman MM, Funderburg NT, Sekaly RP, Klatt NR, Hunt PW (2013) Residual immune dysregulation syndrome in treated HIV infection. Adv Immunol 119: 51-83.

21. Iordache L, Launay O, Bouchaud O, Jeantils V, Goujard C, et al. (2014) Autoimmune diseases in HIV-infected patients: 52 cases and literature review. Autoimmun Rev 13: 850-857.

22. Zandman-Goddard G, Shoenfeld Y (2002) HIV and autoimmunity. Autoimmun Rev 1: 329-337.

23. Stratton R, Slapak G, Mahungu T, Kinloch-de Loes S (2009) Autoimmunity and HIV. Curr Opin Infect Dis 22: 49-56.

24. Brenchley JM, Price DA, Schacker TW, Asher TE, Silvestri G, et al. (2006) Microbial translocation is a cause of systemic immune activation in chronic HIV infection. Nat Med 12: 1365-1371.

25. Krupica T Jr, Fry TJ, Mackall CL (2006) Autoimmunity during lymphopenia: a two-hit model. Clin Immunol 120: 121-128.

26. Jacobs BC, Koga M, van Rijs W, Geleijns K, van Doorn PA, et al. (2008) Subclass IgG to motor gangliosides related to infection and clinical course in Guillain-Barré syndrome. J Neuroimmunol 194: 181-190. 
Citation: Henning F, Bouic P (2014) Increased Frequency of Guillain-Barré Syndrome in HIV Infection: A Prospective Cohort Study. J AIDS Clin Res 5: 332. doi:10.4172/2155-6113.1000332

Page 5 of 5

27. Tsiakalos A, Routsias JG, Kordossis T, Moutsopoulos HM, Tzioufas AG, et al. (2011) Fine epitope specificity of anti-erythropoietin antibodies reveals molecular mimicry with HIV-1 p17 protein: a pathogenetic mechanism for HIV-1-related anemia. J Infect Dis. 204: 902-911.
28. Kanda T, Yamawaki M, Mizusawa H (2003) Sera from Guillain-Barré patients enhance leakage in blood-nerve barrier model. Neurology 60: 301-306.

29. Spindler KR, Hsu TH (2012) Viral disruption of the blood-brain barrier. Trends Microbiol 20: 282-290. 